\title{
Optimal Stochastic Control in Continuous Time with Wiener Processes: General Results and Applications to Optimal Wildlife Management
}

\begin{abstract}
P. Lohmander ${ }^{1}$
We present a stochastic optimal control approach to wildlife management. The objective value is the present value of hunting and meat, reduced by the present value of the costs of plant damages and traffic accidents caused by the wildlife population. First, general optimal control functions and value functions are derived. Then, numerically specified optimal control functions and value functions of relevance to moose management in Sweden are calculated and presented.
\end{abstract}

Keywords: Stochastic optimal control, Wildlife management, Partial differential equations, Moose.

Manuscript was received on 06/08/2017, revised on 23/11/2017 and accepted for publication on 30/11/2017.

\section{Introduction}

We start with a briefing on problem relevant parts of general stochastic optimal control theory, continue with the derivation of general function solutions to the optimal wildlife management problem, and end up with specific derivations and results of relevance to optimal moose management in Sweden.

\section{The General Stochastic Optimal Control Problem}

The general optimal stochastic control methodology in continuous time is briefly presented here. Related introductions with more details are found in Sethi and Thompson [5], Malliaris and Brock [4] and Winston [6]. Lohmander [1] presented connected methods in discrete time. We maximize the objective function,

$$
E\left[\int_{0}^{T} F\left(X_{t}, U_{t}, t\right) d t+S\left(X_{t}, T\right)\right]
$$

where $X_{t}$ is the state variable and $U_{t}$ is the closed loop control variable. Time is represented by $t$ and $T$ is the time horizon. $F($.$) is the instantaneous profit rate and S($.$) is the salvage value. E[$.$] denotes$ expected value. $z_{t}$ is a standard Wiener process.

$$
d X_{t}=f\left(X_{t}, U_{t}, t\right) d t+G\left(X_{t}, U_{t}, t\right) d z_{t}, X_{0}=x_{0}
$$

\footnotetext{
${ }^{1}$ Optimal Solutions in cooperation with Linnaeus University, Umea, Sweden, Email: peter@lohmander.com.
} 
According to the Bellman principle of optimality, we may determine the value function $V(x, t)$ as the maximum of the sum of the net reward during the first short time interval, $F() d$.$t , and the value$ function directly after that time interval:

$$
V(x, t)=\max _{u} E\left[F(x, u, t) d t+V\left(x+d X_{t}, t+d t\right)\right] .
$$

A Taylor approximation gives

$$
V\left(x+d X_{t}, t+d t\right)=V(x, t)+V_{x} d X_{t}+V_{t} d t+\frac{V_{x x}\left(d X_{t}\right)^{2}}{2}+\frac{V_{t t}(d t)^{2}}{2}+V_{x t}\left(d X_{t}\right)(d t)+o(.)
$$

In the Taylor approximation, we need

$$
\left(d X_{t}\right)^{2}=f^{2}(d t)^{2}+2 f G(d t)\left(d z_{t}\right)+G^{2}\left(d z_{t}\right)^{2}
$$

and

$$
d X_{t} d t=f(d t)^{2}+G(d t)\left(d z_{t}\right)
$$

Stochastic calculus tells us

$$
(d t)^{2}=0,(d t)\left(d z_{t}\right)=0,\left(d z_{t}\right)^{2}=d t
$$

Hence, we get

$$
\left(d X_{t}\right)^{2}=G^{2} d t
$$

and

$$
d X_{t} d t=0
$$

Furthermore, $E\left(d z_{t}\right)=0$. As a result, we get

$$
V\left(x+d X_{t}, t+d t\right)=V(x, t)+V_{x} f d t+V_{t} d t+\frac{V_{x x} G^{2}}{2} d t+o(.)
$$

Hence, the value function is approximately given by

$$
V=\max _{u} E\left[F d t+V+V_{x} f d t+V_{t} d t+\frac{V_{x x} G^{2}}{2} d t+o(.)\right]
$$

which leads to 


$$
0=\max _{u} E\left[\left(F+V_{x} f+V_{t}+\frac{V_{x x} G^{2}}{2}+\frac{o(.)}{d t}\right) d t\right] .
$$

With $d t \rightarrow 0$, we have $\frac{o(.)}{d t} \rightarrow 0$, and thus

$$
0=\max _{u} E\left[F+V_{x} f+V_{t}+\frac{V_{x x} G^{2}}{2}\right] .
$$

Since $V_{t}$ is not a function of $u$, we obtain the "Hamilton-Jacobi-Bellman equation" as follows:

$$
-V_{t}=\max _{u} E\left[F+V_{x} f+\frac{V_{x x} G^{2}}{2}\right],
$$

with the boundary condition

$$
V(x, T)=S(x, T)
$$

\section{The Particular Stochastic Optimization Problem}

We want to maximize the expected present value of wildlife management. We need the following notations: $u=u(t)$ is the control variable, the level of hunting at time $t, x=x(t)$ is the size of the wildlife population, $(k, p, f)$ are objective function parameters. The net revenue of the hunting and meat values, $k u-p u^{2}$, is a strictly concave function of the hunting level, $f x$, which is proportional to the population level, $x$, is the cost of destroyed forest plantations and cost of traffic accidents caused by the wildlife population. The population growth increases with the size of the population and decreases with the hunting level. The magnitude of the stochastic population changes depend on the standard Wiener process, $z$, the size of the population, and the risk parameter $s$. With $r$ as the rate of interest in the capital market, we then have the following problem:

$$
\begin{aligned}
& \max E\left(\int_{0}^{\infty} e^{-r t}\left(k u-p u^{2}-f x\right) d t\right) \\
& \text { s.t. } \quad d x=(g x-u) d t+s x d z \\
& \quad k>0, p>0, f>0, s>0 .
\end{aligned}
$$

The net profit at a particular point in time is

$$
R(u, x)=\left(k u-p u^{2}-f x\right) .
$$

The "Hamilton-Jacobi-Bellman equation" becomes 


$$
-J_{t}(x, t)=e^{-r t} R(u(t), x(t))+\frac{s^{2} x^{2} J_{x x}(x, t)}{2}+J_{x}(x, t)(g x(t)-u(t)) .
$$

Now, the problem is to determine the value function and the control function so that the HamiltonJacobi-Bellman equation (HJBE) is satisfied. Let us assume that the value function can be expressed as follows:

$$
\begin{gathered}
V(x)=a+b x+c x^{2}, \\
J(x(t), t)=e^{-r t} V(x)=e^{-r t}\left(a+b x+c x^{2}\right) .
\end{gathered}
$$

Then, by differentiation, we get

$$
\begin{gathered}
J_{x}(x(t), t)=e^{-r t}(b+2 c x), \\
J_{x x}(x(t), t)=e^{-r t}(2 c), \\
J_{t}(x(t), t)=-r e^{-r t}\left(a+b x+c x^{2}\right) .
\end{gathered}
$$

As a result, we can rewrite $\mathrm{HJBE}$ as follows:

$$
\begin{gathered}
r V(x)=R(u, x)+\frac{s^{2} x^{2} V_{x x}(x)}{2}+V_{x}(x)(g x-u), \\
r\left(a+b x+c x^{2}\right)=\left(k u-p u^{2}-f x\right)+\frac{1}{2} s^{2} x^{2} 2 c+(b+2 c x)(g x-u) .
\end{gathered}
$$

We need to optimize the control, $u$ :

$$
\max _{u} Z(u)=\left(k u-p u^{2}-f x\right)+\frac{1}{2} s^{2} x^{2} 2 c+(b+2 c x)(g x-u) .
$$

The first order and second order maximal conditions are

$$
\begin{gathered}
\frac{d Z(u)}{d u}=k-2 p u-b-2 c x=0, \\
\frac{d^{2} Z(u)}{d u^{2}}=-2 p<0 .
\end{gathered}
$$

The derived value of $u$ is a unique maximum, $u^{*}$, which is obtained to be

$$
\begin{gathered}
\left(\frac{d Z(u)}{d u}=0\right) \Rightarrow\left(u=u^{*}=\frac{k-b-2 c x}{2 p}\right), \\
Z^{*}=Z\left(u^{*}\right)=\left(k u^{*}-p\left(u^{*}\right)^{2}-f x\right)+\frac{1}{2} s^{2} x^{2} 2 c+(b+2 c x)\left(g x-u^{*}\right),
\end{gathered}
$$




$$
Z^{*}=(k-b-2 c x) u^{*}-p\left(u^{*}\right)^{2}-f x+\frac{1}{2} s^{2} x^{2} 2 c+b g x+2 c g x^{2} .
$$

Using the optimal values of the control, via the optimized control function, we get

$$
Z^{*}=(k-b-2 c x)\left(\frac{k-b-2 c x}{2 p}\right)-p\left(\frac{k-b-2 c x}{2 p}\right)^{2}-f x+\frac{1}{2} s^{2} x^{2} 2 c+b g x+2 c g x^{2} .
$$

The HJBE can now be written as

$$
\begin{gathered}
r V(x)=R\left(u^{*}, x\right)+\frac{s^{2} x^{2} V_{x x}(x)}{2}+V_{x}(x)\left(g x-u^{*}\right) \\
r\left(a+b x+c x^{2}\right)=\left(k u^{*}-p\left(u^{*}\right)^{2}-f x\right)+\frac{1}{2} s^{2} x^{2} 2 c+(b+2 c x)\left(g x-u^{*}\right) \\
r\left(a+b x+c x^{2}\right)=(k-b-2 c x)\left(\frac{k-b-2 c x}{2 p}\right)-p\left(\frac{k-b-2 c x}{2 p}\right)^{2}-f x \\
r a+r b x+r c x^{2}=\frac{1}{2 p}(k-b-2 c x)^{2}-\frac{1}{4 p}(k-b-2 c x)^{2}-f x+\frac{1}{2} s^{2} x^{2} 2 c+b g x+2 c g x^{2} \\
r a+r b x+r c x^{2}=\frac{1}{4 p}(k-b-2 c x)^{2}-f x+\frac{1}{2} s^{2} x^{2} 2 c+b g x+2 c g x^{2} \\
r a+r b x+r c x^{2}=\frac{1}{4 p}\left(k^{2}+b^{2}+4 c^{2} x^{2}-2 b k-4 c k x+4 b c x\right)-f x+\frac{1}{2} s^{2} x^{2} 2 c+b g x \\
+2 c g x^{2} \\
r a+r b x+r c x^{2}=\frac{1}{4 p}\left(k^{2}+b^{2}-2 b k-4 c k x+4 b c x+4 c^{2} x^{2}\right)-f x+c s^{2} x^{2}+b g x+2 c g x^{2} \\
r a+r b x+r c x^{2}=\frac{k^{2}+b^{2}-2 b k}{4 p}+\frac{c(b-k)}{p} x+\frac{c^{2}}{p} x^{2}-f x+c s^{2} x^{2}+b g x+2 c g x^{2} .
\end{gathered}
$$

Now, we have obtained a quadratic function, that always has to be zero. If the function is notzero, then the HJBE is violated. Since the function must hold for all possible values of $x$, the size of the population, it is clear that we have three equations that can be used to determine the parameters $(a, b, c)$ :

$$
0=\left(\frac{k^{2}+b^{2}-2 b k}{4 p}-r a\right)+\left(\frac{c(b-k)}{p}+b g-r b-f\right) x+\left(\frac{c^{2}}{p}+c s^{2}+2 c g-r c\right) x^{2} .
$$

First, the parameter $c$ is determined by solving 


$$
\left[0=\left(\frac{c^{2}}{p}+c s^{2}+2 c g-r c\right) x^{2}, x \neq 0\right]
$$

Which leads to

$$
\frac{c^{2}}{p}+c s^{2}+2 c g-r c=0 \Rightarrow \frac{c}{p}+s^{2}+2 g-r=0 \Rightarrow c=p\left(r-2 g-s^{2}\right) .
$$

Next, the parameter $b$ is determined as follows:

$$
\begin{aligned}
& {\left[0=\left(\frac{c(b-k)}{p}+b g-r b-f\right) x \quad, x \neq 0\right] \Rightarrow} \\
& \frac{c(b-k)}{p}+b g-r b-f=0 \Rightarrow\left(\frac{c}{p}+g-r\right) b-\frac{c k}{p}-f=0 \Rightarrow \\
& b=\frac{\frac{c k}{p}+f}{\frac{c}{p}+g-r} \Rightarrow b=\frac{c k+p f}{c+p(g-r)},
\end{aligned}
$$

and thus, by substituting for $\mathrm{c}$, we get

$$
\begin{aligned}
& b=\frac{p\left(r-2 g-s^{2}\right) k+p f}{p\left(r-2 g-s^{2}\right)+p(g-r)} \Rightarrow b=\frac{\left(r-2 g-s^{2}\right) k+f}{\left(r-2 g-s^{2}\right)+(g-r)} \Rightarrow \\
& b=\frac{\left(r-2 g-s^{2}\right) k+f}{-g-s^{2}} \Rightarrow b=\frac{k\left(2 g-r+s^{2}\right)-f}{g+s^{2}} .
\end{aligned}
$$

Finally, the parameter $a$ is determined:

$$
\begin{aligned}
& {\left[0=\left(\frac{k^{2}+b^{2}-2 b k}{4 p}-r a\right)\right] \Rightarrow \frac{k^{2}+b^{2}-2 b k}{4 p}-r a=0 \Rightarrow} \\
& k^{2}+b^{2}-2 b k=4 p r a \Rightarrow(k-b)^{2}=4 p r a \Rightarrow a=\frac{(k-b)^{2}}{4 p r} .
\end{aligned}
$$

Now, the expression for $b$ can be used in the expression for $a$ to get 


$$
\begin{aligned}
& a=\frac{\left(k-\frac{k\left(2 g-r+s^{2}\right)-f}{g+s^{2}}\right)^{2}}{4 p r} \Rightarrow a=\frac{\left(k\left(g+s^{2}\right)-k\left(2 g-r+s^{2}\right)+f\right)^{2}}{4 p r\left(g+s^{2}\right)^{2}} \Rightarrow \\
& a=\frac{\left(f+k g+k s^{2}-2 k g+k r-k s^{2}\right)^{2}}{4 p r\left(g+s^{2}\right)^{2}} \Rightarrow a=\frac{(f-k(g-r))^{2}}{4 p r\left(g+s^{2}\right)^{2}} .
\end{aligned}
$$

Now, we know the parameters of the value function. They are explicit functions of the parameters in the initially specified optimization problem:

$$
\begin{gathered}
V(x)=a+b x+c x^{2}, \\
V(x)=\frac{(f-k(g-r))^{2}}{4 p r\left(g+s^{2}\right)^{2}}+\left(\frac{k\left(2 g-r+s^{2}\right)-f}{g+s^{2}}\right) x+p\left(r-2 g-s^{2}\right) x^{2} .
\end{gathered}
$$

Next, we will determine the optimal control function. We know

$$
u^{*}=\left(\frac{k-b-2 c x}{2 p}\right)
$$

By substituting for $b$ and $c$, we get

$$
\begin{gathered}
u^{*}=\left(\frac{k-\left(\frac{k\left(2 g-r+s^{2}\right)-f}{g+s^{2}}\right)-2 p\left(r-2 g-s^{2}\right) x}{2 p}\right) \\
u^{*}=\left(\frac{k-\left(\frac{k\left(2 g-r+s^{2}\right)-f}{g+s^{2}}\right)+2 p\left(2 g-r+s^{2}\right) x}{2 p}\right) \Rightarrow \\
u^{*}=\left(\frac{k-\left(\frac{k\left(2 g-r+s^{2}\right)-f}{g+s^{2}}\right)}{2 p}+\left(2 g-r+s^{2}\right) x\right) \Rightarrow \\
u^{*}=\frac{k-\left(\frac{k\left(2 g-r+s^{2}\right)-f}{g+s^{2}}\right)}{2 p}+\left(2 g-r+s^{2}\right) x \Rightarrow
\end{gathered}
$$




$$
\begin{aligned}
& u^{*}=\frac{k\left(g+s^{2}\right)-\left(k\left(2 g-r+s^{2}\right)-f\right)}{2 p\left(g+s^{2}\right)}+\left(2 g-r+s^{2}\right) x \Rightarrow \\
& u^{*}=\frac{k g+k s^{2}-2 k g+k r-k s^{2}+f}{2 p\left(g+s^{2}\right)}+\left(2 g-r+s^{2}\right) x \Rightarrow \\
& u^{*}=\frac{k(r-g)+f}{2 p\left(g+s^{2}\right)}+\left(2 g-r+s^{2}\right) x .
\end{aligned}
$$

Now, we know that the optimal control is a linear function of the population level. The intercept and the slope of this linear control function are explicit functions of the initially specified parameters.

\section{The Numerically Specified Case}

Let us enter the particular numerical parameters of a real problem. Lohmander [2] estimated revenue and cost functions and calculated the optimal equilibrium moose population in Sweden, under deterministic assumptions and no discounting. However, the size of the moose population is not exactly predictable and the capital market often, but not always, includes strictly positive interest rates. Random disturbances can have large effects. We may determine the optimal stochastic control of the moose population in Sweden, based on the new general functions derived here. The figures and functions presented in Lohmander [2] and [3] can be used to derive the parameters of the stochastic control problem. Note that the quadratic objective function in the stochastic optimal control problem here is an approximation of the particular objective function presented in [2] and [3]. Both functions are strictly concave. The quadratic approximation fits the original function very well within the selected approximation region. Of course, the derived general equations can also be used for other animals and in other countries of the world. These parameter values were estimated as $g=1 / 3$, $k=600, p=90, f=90$. Let $r=1 / 30$. In Fig. 1. and Fig. 2, we can inspect the optimal value function and the optimal control function.

Fig. 1 shows that the optimal value function is a strictly concave function of the size of the population. The value is a decreasing function of the stochastic parameter $s$. The optimal population density, with respect to the value function, is a decreasing function of $s$. Note that the optimal population density, with respect to the value function, is not equal to the optimal population density in the long run. The value may be high with a rather high (initial) population, since the control (hunting level) initially can be high, gradually reducing the population to a much lower level. Fig. 2 shows the optimal control, the hunting level, as a function of the size of the population. Note that the optimal control level is an increasing function of the stochastic parameter $s$. The intersections of the alternative control functions and the line representing "expected population growth without hunting", show the population levels and the hunting levels where the expected values of the instant population changes are zero. Observe that, if $s$ increases, these "expected equilibria" intersections obtain lower population densities and lower hunting levels. Since the first derivatives of the alternative control functions with respect to $x$ are higher than the first derivatives of "the expected population growth without hunting", with respect to $x$, the "expected equilibria" are dynamically stable. 


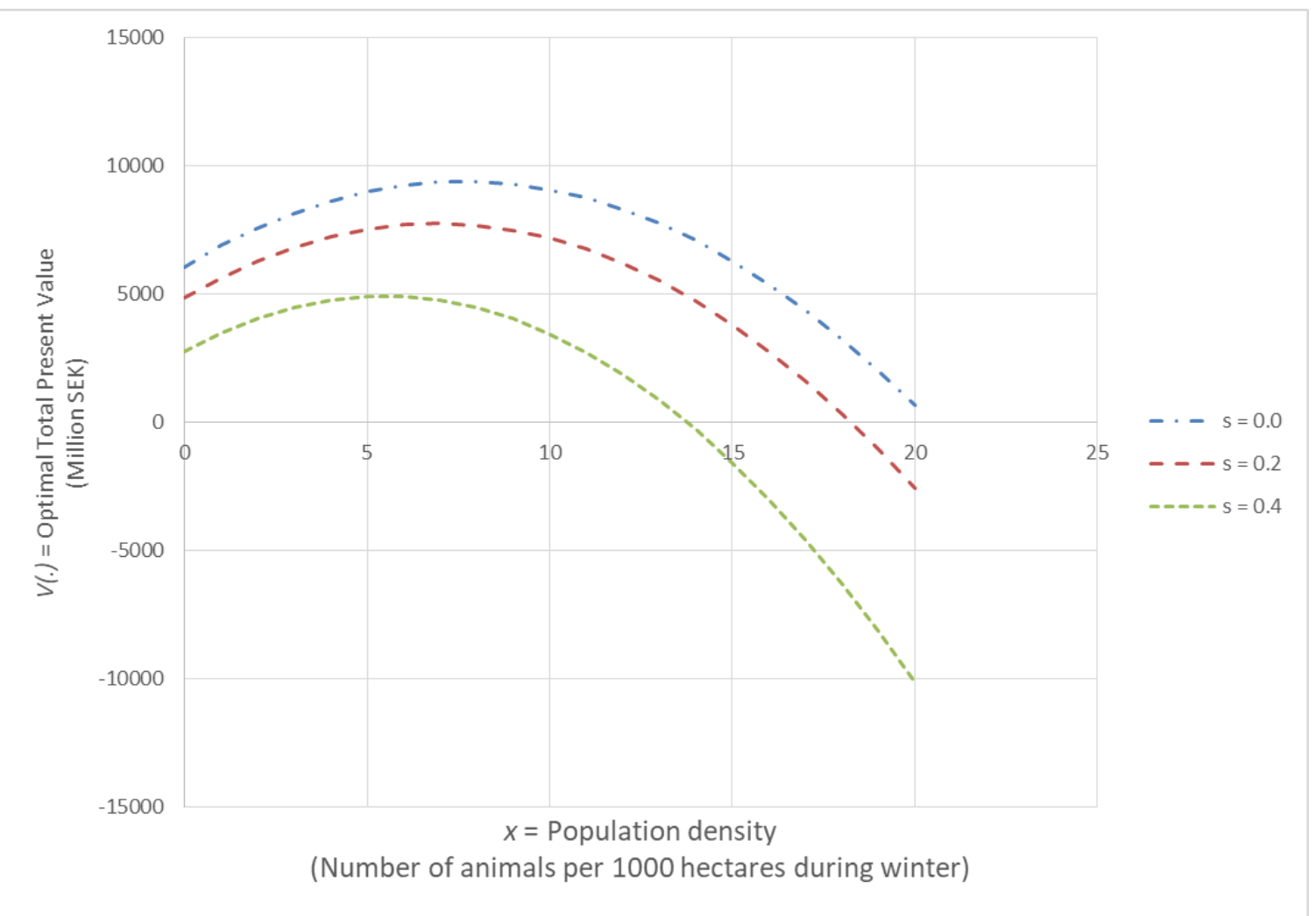

Figure 1. The optimal total present value function, $V($.$) as a function of the population density, x$, and the stochastic parameter $s$.

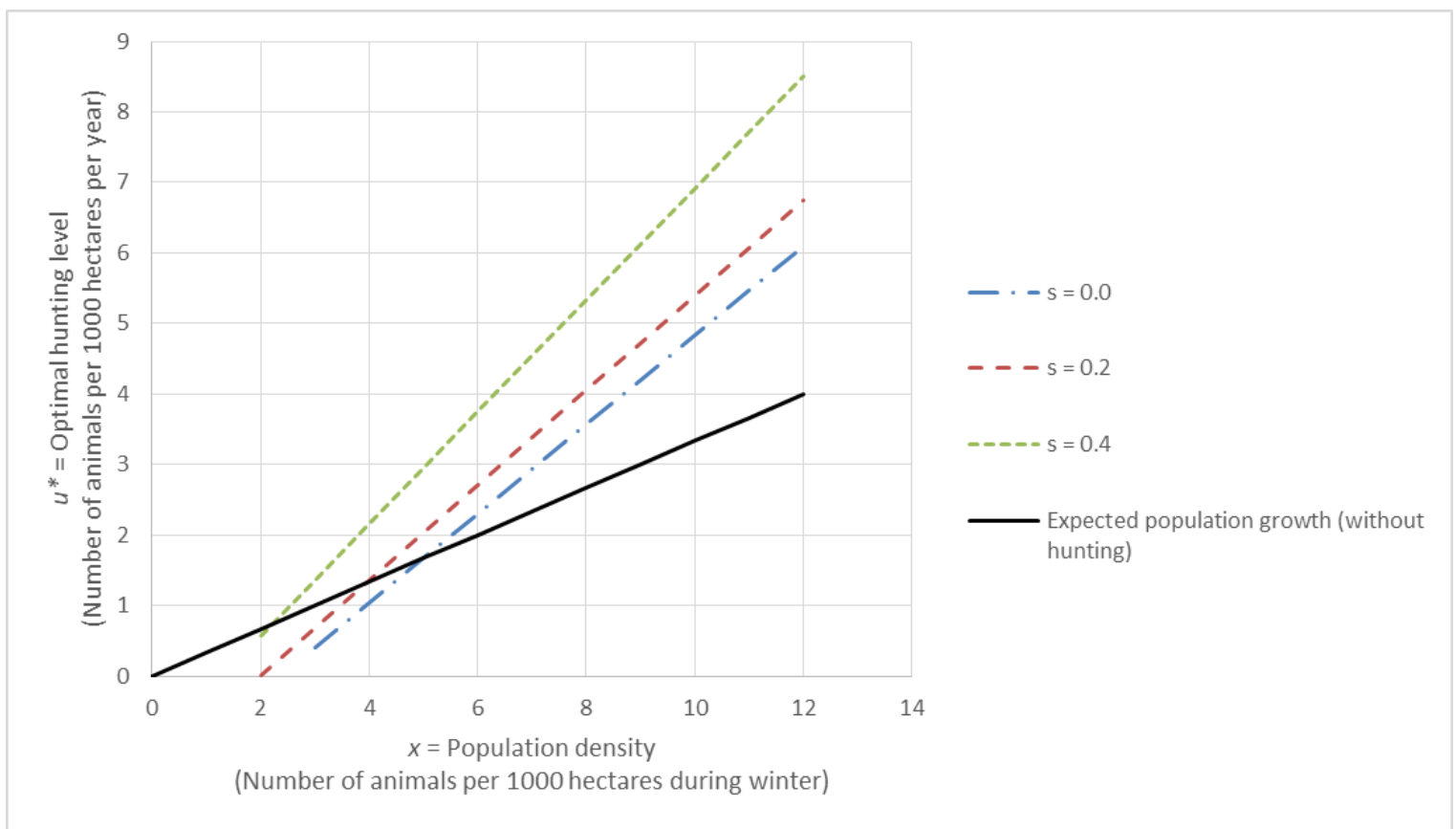

Figure 2. The optimal control, the hunting level, $u^{*}$, as a function of the population density, $x$, and the stochastic parameter $s$. 


\section{Conclusion}

We has derived and presented a stochastic optimal control approach to wildlife management. The objective value was the net present value of hunting and meat, reduced by the present value of the costs of plant damages and traffic accidents caused by the wildlife population. General optimal control functions and value functions were derived. Then, numerically specified optimal control functions and value functions of relevance to moose management in Sweden were calculated.

\section{Acknowledgement}

The author appreciates travel grants from FORMAS.

\section{References}

[1] Lohmander, P., (2007), Adaptive Optimization of Forest Management in a Stochastic World, in Weintraub A. et al (Ed), Handbook of Operations Research in Natural Resources, Springer, Springer Science, International Series in Operations Research and Management Science, New York, USA, 525-544.

[2] Lohmander, P., (2011), Hur många älgar har vi råd med? Vi Skogsägare, Debatt, Nr 1.

[3] Lohmander, P., (2011), Älgens ekonomi och den ekonomiskt optimala älgstammen, Skogen och Viltet, SLU, Umea, November 24, http://www.lohmander.com/LohmanderSkogenochViltet2011.pdf

[4] Malliaris, A.G., and Brock, W.A., (1984), Stochastic Methods in Economics and Finance, North-Holland, Amsterdam.

[5] Sethi, S.P., and G.L.Thompson, G.L., (2000), Optimal Control Theory, Applications to Management Science and Economics, Kluwer Academic Publishers, Boston.

[6] Winston, W.L., (2004), Operations Research: Volume Two, Introduction to Probability Models, Thomson Brooks/Cole, 4 ed. 